\title{
Predictors and outcomes of resistant hypertension among patients with coronary artery disease and hypertension
}

\author{
Steven M. Smith ${ }^{a}$, Yan Gong ${ }^{b}$, Eileen Handberg ${ }^{c}$, Franz H. Messerli ${ }^{d}$, George L. Bakris ${ }^{e}$, Ali \\ Ahmed $^{\dagger}$, Anthony A. Bavry ${ }^{c}$, Carl J. Pepine ${ }^{c}$, and Rhonda M. Cooper-DeHoff ${ }^{b, c}$ \\ aDepartment of Clinical Pharmacy, Skaggs School of Pharmacy and Pharmaceutical Sciences, \\ University of Colorado, Aurora, Colorado \\ bepartment of Pharmacotherapy and Translational Research, College of Pharmacy, University \\ of Florida, Gainesville, Florida \\ 'Division of Cardiovascular Medicine, Department of Medicine, College of Medicine, University of \\ Florida, Gainesville, Florida \\ dDivision of Cardiology, St. Luke's-Roosevelt Hospital, New York \\ eDepartment of Preventive Medicine, Rush University, Chicago, Illinois \\ fDepart-Departments of Medicine and Epidemiology, Schools of Medicine and Public Health, \\ University of Alabama Birmingham, Birmingham, Alabama, USA
}

\begin{abstract}
Objective-Resistant hypertension (res-HTN) is a challenging problem, but little is known of res-HTN in patients with coronary artery disease (CAD). In this post-hoc INternational VErapamil SR-Trandolapril STudy (INVEST) analysis, we assessed prevalence, predictors, and impact on outcomes of res-HTN in CAD patients with hypertension.
\end{abstract}

Methods-Participants ( $n=17$ 190) were divided into three groups according to achieved blood pressure (BP): controlled (BP <140/90 $\mathrm{mmHg}$ on three or fewer drugs); uncontrolled (BP $\geq 140 / 90 \mathrm{mmHg}$ on two or fewer drugs); or resistant (BP $\geq 140 / 90 \mathrm{mmHg}$ on three drugs or any patient on at least four drugs).

Results-The prevalence of res-HTN was 38\%: significant predictors of res-HTN included heart failure [odds ratio (OR) 1.73], diabetes (OR 1.63), Black race (OR 1.50), and US residence (OR 1.50). Compared with controlled HTN, res-HTN had multivariate-adjusted association with higher risk of adverse outcomes \{first occurrence of all-cause death, nonfatal myocardial infarction, or nonfatal stroke [hazard ratio 1.27, 95\% confidence interval (CI) 1.13-1.43], and individual outcomes of all-cause death (hazard ratio $1.29,95 \%$ CI 1.13-1.48), cardiovascular mortality (hazard ratio $1.47,95 \%$ CI 1.21-1.78), and nonfatal stroke (hazard ratio 1.61, 95\% CI 1.17-2.22),

(C) 2014 Wolters Kluwer Health | Lippincott Williams \& Wilkins.

Correspondence to Rhonda M. Cooper-DeHoff, PharmD, MS, Associate Professor, Department of Pharmacotherapy and Translational Research and Center for Pharmacogenomics, University of Florida, Gainesville, FL 32610, USA. Tel: +1 352 273 6184, fax: +1 352 273 6121, dehoff@cop.ufl.edu.

Conflicts of interest: There are no conflicts of interest. 
but not nonfatal myocardial infarction (hazard ratio 0.98 , 95\% CI $0.72-1.34$ ) \}. Adverse outcomes, except nonfatal stroke, did not differ in patients with res-HTN compared to uncontrolled HTN.

Conclusions-Res-HTN is common in patients with CAD and hypertension, associated with poor prognosis, and linked with a number of conditions. Emphasis should be placed on recognizing those at risk for res-HTN and future studies should examine whether more aggressive treatment of res-HTN improves outcomes.

\section{Keywords}

blood pressure; coronary artery disease; hypertension; INVEST; resistant hypertension

\section{Introduction}

Treatment-resistant hypertension (res-HTN) - requiring at least four antihypertensive agents for blood pressure (BP) control - is a challenging clinical problem [1]. Clinical trial data suggest that res-HTN is present in 20-30\% of patients with hypertension [2,3]. Analyses of epidemiologic data generally support these clinical trial estimates and further suggest that the prevalence has more than doubled over the past quarter century [4-7]. Importantly, resHTN has been associated with a 1.1-3-fold increase in the risk of cardiovascular events compared with treatment-responsive HTN in cohort studies [8-10].

Although res-HTN has come under increased focus in recent years, there remains a paucity of data regarding the impact of res-HTN on adverse cardiovascular outcomes in patients with documented coronary artery disease (CAD). Ischemic heart disease, in particular, is the leading cause of mortality globally and is more common in patients with res-HTN than those with treatment-responsive HTN $[2,11]$. Therefore, the aim of this study was to describe the prevalence, predictors, and impact on adverse cardiovascular outcomes of res-HTN among patients with CAD and HTN. We hypothesized that res-HTN would portend increased risk for cardiovascular outcomes. To test this hypothesis, we used data from the INternational VErapamil-trandolapril STudy (INVEST), which evaluated two antihypertensive treatment regimens in a population of patients with CAD and HTN.

\section{Methods}

\section{Study design}

The INVEST design, methods, and principal results have been previously published [12,13]. Briefly, this was a prospective, randomized, open-label, blinded endpoint trial that compared clinical outcomes in 22576 participants with CAD and HTN who were randomly assigned to a calcium antagonist-based (verapamil SR \pm trandolapril) or $\beta$-blocker-based (atenolol \pm hydrochlorothiazide) antihypertensive treatment strategy. Nonstudy antihypertensive medications were allowable except for $\beta$-blockers in the calcium antagonist arm and calcium antagonists in the $\beta$-blocker arm. Participants were aged at least 50 years with HTN and clinically stable CAD. 


\section{Definition of resistant hypertension and assembly of study cohort}

To examine the impact of res-HTN, participants were grouped according to achieved HTN control based on the BP at the visit immediately prior to an event or censoring, according to the following: controlled HTN, defined as BP below 140/90 $\mathrm{mmHg}$ on three or fewer antihypertensive drugs; uncontrolled HTN, defined as BP at least 140/90 mmHg on two or fewer antihypertensive drugs; res-HTN, defined as BP at least 140/90 $\mathrm{mmHg}$ on at least three antihypertensive drugs or BP below $140 / 90 \mathrm{mmHg}$ on at least four antihypertensive drugs (Fig. 1) [1,14]. In secondary analyses, participants with res-HTN were further subdivided into controlled res-HTN (BP $<140 / 90 \mathrm{mmHg}$ on at least four antihypertensive agents) and uncontrolled res-HTN (BP $\geq 140 / 90 \mathrm{mmHg}$ on at least three antihypertensive agents). Finally, sensitivity analyses were performed in which res-HTN was defined more narrowly as BP at least 140/90 $\mathrm{mmHg}$ on at least three antihypertensive drugs including a diuretic, or BP below 140/90 $\mathrm{mmHg}$ on at least four antihypertensive drugs including a diuretic. Accordingly, in these sensitivity analyses, those who were not taking a diuretic were excluded from the res-HTN group and instead classified as controlled (BP $<140 / 90$ $\mathrm{mmHg}$ ) or uncontrolled (BP $\geq 140 / 90 \mathrm{mmHg}$ ), regardless of the total number of antihypertensive drugs being used. Participants with SBP below $130 \mathrm{mmHg}$ and DBP below $80 \mathrm{mmHg}(n=5386)$ were excluded from all analyses since this threshold has previously been explored and determined to be associated with worse cardiovascular outcomes (except stroke) and all-cause mortality [15].

\section{Outcomes}

The primary outcome for this analysis was first occurrence of all-cause mortality, nonfatal myocardial infarction (MI), or nonfatal stroke [12]. Secondary outcomes included the individual components of the primary outcome as well as cardiovascular mortality. Overall, both treatment strategies achieved excellent BP control (>70\% with both strategies had BP $<140 / 90 \mathrm{mmHg}$ ) and were equally effective in reducing adverse outcomes [12].

\section{Statistical analyses}

Because we previously demonstrated that the antihypertensive treatment strategies had equivalent impact on the outcomes and very similar effects on SBP and DBP [12], all patients were pooled, regardless of initial treatment assignment. For the primary, secondary, and sensitivity analyses, univariate and multivariate adjusted Cox proportional hazards models were used to investigate relationships between HTN control group and the adverse outcomes. The reference for all comparisons between groups was the controlled HTN group. Baseline predictors of res-HTN were assessed using multivariate logistic regression models. For both the Cox and logistic regression models, a stepwise approach was used with a cutoff of $P$ less than 0.2 for entry into the model and a threshold of $P$ less than 0.05 for remaining in the final model. To further investigate risk of developing res-HTN, a risk factor construct was developed using independent baseline predictors of res-HTN identified in logistic regression models. Finally, descriptive statistics were used for baseline patient characteristics, and differences in these characteristics between HTN control groups were tested using analysis of variance for continuous variables and chi-square test for categorical variables. 
For all analyses, a $P$ value less than 0.05 was considered statistically significant. Statistical analyses were performed using SAS version 9.3 (SAS Institute, Cary, North Carolina, USA).

\section{Results}

After exclusion of patients with BP below 130/80 mmHg, a total of 17190 INVEST participants were included in this analysis (Fig. 1). Interestingly, all of the participants excluded from these analyses on the basis of BP below 130/80 $\mathrm{mmHg}$ were taking three or less antihypertensive agents and thus would not meet the definition of res-HTN. Of the participants included in this analysis, 7615 (44.3\%) had controlled HTN (BP <140/90 mmHg), 3085 (17.9\%) had uncontrolled HTN, and 6490 (37.8\%) had res-HTN. A total of 46 190 patient-years of follow-up were accumulated.

Baseline characteristics according to HTN group are summarized in Table 1. On average, patients with res-HTN were older, had a higher baseline SBP, and were more likely to be Black. Patients with res-HTN also had a greater baseline prevalence of history of MI, stroke, heart failure, diabetes, renal impairment, and other comorbidities. Baseline use of lipidlowering agents, aspirin, potassium supplements, and NSAIDs was also more prevalent in the res-HTN group.

\section{On-treatment blood pressure and medication use}

Mean (SD) on-treatment SBP for the visit immediately prior to an event or censoring was 143 (15)mmHg for the res-HTN group, compared with 132 (9) $\mathrm{mmHg}$ for the controlled HTN group, and 144 (15) mmHg for the uncontrolled HTN group $(P<0.0001)$. Corresponding mean (SD) on-treatment DBP was 81 (9) for the res-HTN group, compared with 79 (6) for the controlled HTN group, and 82 (9) for the uncontrolled HTN group $(P<0.0001)$. Figure 2 displays mean SBP and DBP according to HTN control status during follow-up. Mean SBP and DBP over the entire study period were not significantly different from mean SBP and DBP at the visit prior to an event or censoring (data not shown).

The total number of antihypertensive drugs used at the last visit or visit immediately preceding an event or censoring are summarized in Fig. 3, according to HTN control group. Data regarding study drug use and median doses are summarized in Table 2. The median daily doses of verapamil SR and atenolol were higher in the res-HTN group (verapamil SR, $360 \mathrm{mg}$; atenolol, $100 \mathrm{mg}$ ) compared with both the controlled and uncontrolled HTN groups (verapamil SR, $240 \mathrm{mg}$; atenolol, $50 \mathrm{mg} ; P<0.0001$ for comparison of res-HTN group to both controlled and uncontrolled HTN groups for both drugs). In contrast, the median doses of trandolapril (4 mg) and hydrochlor-othiazide $(25 \mathrm{mg}$ ) were not different comparing the HTN groups.

\section{Baseline predictors of risk for resistant hypertension}

In the multivariate logistic regression model, history of heart failure [odds ratio (OR) 1.73, 95\% confidence interval (CI) 1.50-1.99], diabetes (OR 1.63, 95\% CI 1.52-1.74), or renal insufficiency (OR 1.34, 95\% CI 1.06-1.70), US residence (OR 1.50, 95\% CI 1.38-1.63) and Black vs. White race (OR 1.50, 95\% CI 1.36-1.65) were characteristics associated with increased risk for res-HTN. In sum, we found 13 independent predictors of res-HTN (Fig. 
4). The overall prevalence of res-HTN increased as the number of risk factors present increased, ranging from $25.6 \%$ in participants with no risk factors to $78.6 \%$ in participants with at least eight risk factors $(P<0.0001$ for trend; Fig. 5).

\section{Cardiovascular and mortality outcomes}

The primary adverse outcome of first occurrence of all-cause mortality, nonfatal MI, or nonfatal stroke occurred in 1724 (10.03\%) participants overall, including 524 (6.9\%) events in the controlled HTN group, $354(11.5 \%)$ in the uncontrolled group, and $846(13.0 \%)$ in the res-HTN group (Fig. 6). Compared with the controlled HTN group, the unadjusted hazard ratios for the occurrence of the primary adverse outcome in patients with res-HTN and uncontrolled HTN were 1.84 (95\% CI 1.65-2.05, $P<0.0001$ ) and 1.67 (95\% CI 1.46-1.91, $P<0.0001)$, respectively. The primary adverse outcome was significantly different comparing the three groups (overall log-rank $P<0.0001$; Fig. 7). However, there was no difference between the uncontrolled and res-HTN groups (log-rank $P=0.11)$. In the multivariate-adjusted analyses, patients in the res-HTN group, compared with the controlled HTN group, had a higher risk of the primary adverse outcome (hazard ratio 1.27; 95\% CI $1.13-1.43, P<0.0001)$ and secondary outcomes, including all-cause mortality (hazard ratio $1.29,95 \%$ CI $1.13-1.48, P=0.0002$ ), cardiovascular mortality (hazard ratio $1.47,95 \%$ CI $1.21-1.78, P<0.0001$ ), and nonfatal stroke (hazard ratio $1.61,95 \%$ CI 1.17-2.22, $P=0.0035$ ), but not nonfatal MI (hazard ratio $0.98,95 \%$ CI $0.72-1.34, P=0.91$; Fig. 6). Comparing the res-HTN and uncontrolled HTN groups, the only outcome that differed was nonfatal stroke (hazard ratio $1.52,95 \%$ CI 1.07-2.15, $P=0.02$; Fig. 6).

In the sensitivity analyses, in which res-HTN classification required use of a diuretic, we observed no appreciable difference in the primary adverse outcome or any of the secondary outcomes (see Supplemental Table, http://links.lww.com/HJH/A302).

\section{Outcomes in controlled vs. uncontrolled resistant hypertension}

As expected, the patients with controlled res-HTN had lower mean BP compared to patients with uncontrolled res-HTN at baseline ( $148 / 85$ vs. $157 / 88 \mathrm{mmHg}$, respectively; $P<0.0001$ for both SBP and DBP comparisons) and at the visit prior to an event or censoring (125/74 vs. $153 / 84 \mathrm{mmHg}$, respectively; $P<0.0001$ for both comparisons). However, no significant differences were found between res-HTN groups with regard to any adverse outcomes (data not shown).

\section{Discussion}

The prevalence of res-HTN has increased substantially in recent decades and observational studies have shown that res-HTN is associated with an increased risk of cardiovascular events as compared with nonresistant HTN [4,8,9]. Our results confirm these findings and extend them to patients with concomitant CAD and HTN that were well managed. To our knowledge, this is the first study examining the impact of res-HTN on clinical outcomes in a population of patients with CAD and HTN. Interestingly, we observed that res-HTN occurred in over a third of patients included in the present analysis. Furthermore, patients classified as having res-HTN, compared with those with controlled HTN, were at a 
significantly increased risk of all-cause mortality, cardiovascular mortality, nonfatal stroke, and the first occurrence of all-cause mortality, non-fatal MI, or nonfatal stroke.

Precise estimates of the prevalence of res-HTN are difficult to determine as evidenced by the wide range (12-46\%) reported in observational studies and clinical trials $[2,4,6,8]$. This imprecision is due, at least in part, to the variety of hypertensive populations studied and the variety of treatment algorithms used. However, a recent registry report suggested that resHTN [using American Heart Association (AHA) definition] was prevalent in a fifth of patients with either at least three atherosclerotic risk factors or established disease [10]. In the present study, we observed a higher res-HTN prevalence of approximately $38 \%$ of patients with HTN and CAD.

Among INVEST participants, we found that the presence of cardiovascular-related comorbidities [heart failure and left-ventricular hypertrophy (LVH), diabetes, history of stroke/transient ischemic attack (TIA), percutaneous coronary intervention (PCI), and peripheral vascular disease (PVD)], renal insufficiency, Black race, and US residency were the strongest predictors of res-HTN. Thus, it seems reasonable that these patients with resHTN had more severe vascular disease in general. Interestingly, all ORs were less than 2, suggesting modest independent associations for any single risk factor. However, the prevalence of res-HTN was at least $50 \%$ in participants with five or more risk factors (nearly $20 \%$ of the study population). In addition, increasing age, increasing BMI, and female sex were all associated with classification as res-HTN. These findings are similar to those from the Antihypertensive and Lipid-Lowering Treatment to Prevent Heart Attack Trial (ALLHAT) in HTN patients with high cardiovascular risk, but not necessarily established cardiovascular disease [2]. In addition, our findings are consistent with those of the Avoiding Cardiovascular events through COMbination therapy in Patients LIving with Systolic Hypertension (ACCOMPLISH) sub-study of people with CAD that noted older people and those with chronic kidney disease require more antihypertensive medications to achieve BP control [16]. However, our results differ in some respects from the AngloScandinavian Cardiac Outcomes Trial (ASCOT), in which age was not predictive of resistant HTN (although patients aged $\geq 80$ years were excluded), female sex was protective, and Whites had a greater risk of developing res-HTN (although 98.6\% of participants were white) [3]. Regardless, our findings suggest that risk factors for res-HTN appear comparable in patients with HTN with and without established cardiovascular disease and underscore the need to recognize patients with CAD whom are at risk for res-HTN.

Interestingly, treatment with the $\beta$-blocker strategy was associated with greater risk of resHTN compared with the calcium antagonist strategy, despite similar mean BP reduction [12]. This finding is consistent with data from ASCOT, in which participants assigned to the calcium antagonist strategy had a lower risk of res-HTN development compared with those assigned to the $\beta$-blocker strategy [3]. One possible explanation for this finding is the differential effects of $\beta$-blockers vs. other antihypertensive agents on central hemodynamics and aortic stiffness. Atenolol, in particular, appears to be inferior to other antihypertensives in reducing central BP and improving arterial stiffness parameters [17]. In addition, in INVEST, participants assigned to the calcium antagonist strategy, compared with the $\beta$ blocker strategy, used trandolapril more frequently (63 vs. 52\%, respectively, at 24 months) 
and hydrochlorothiazide less frequently (44 vs. 60\%, respectively, at 24 months). Angiotensin-converting enzyme (ACE) inhibitors have demonstrated consistent improvements in arterial stiffness parameters, whereas hydrochlorothiazide seems to have negligible effects on arterial stiffness [17-20]. Alternatively, the proclivity for $\beta$-blockers to increase res-HTN risk may be related to the fact that $\beta_{1}$-selective adrenergic antagonists suppress melatonin synthesis in the pineal gland, which may, in turn, alter circadian rhythm, raise $\mathrm{BP}$, and increase sympathetic nervous system activity [21-23].

Perhaps the most salient finding from our study is that res-HTN portends increased risk of major cardiovascular events and all-cause mortality compared with controlled HTN. These results support findings from two recently published studies using data from the

Cardiovascular Research Network hypertension registry [9] and the REACH registry [10], in which res-HTN was associated with poorer prognosis than nonresistant HTN. In the Cardiovascular Research Network registry, using data from 18036 patients with incident HTN, the development of res-HTN, as compared to nonresistant HTN, was associated with a non-significantly higher incidence of death ( 2.1 vs. $1.9 \%$, respectively), stroke ( 0.6 vs. $0.5 \%)$, and heart failure (0.4 vs. $0.3 \%)$, but not MI (0.4 vs. $0.5 \%)$ [9]. An analysis of the composite of these endpoints revealed a trend towards increased risk of adverse outcomes in patients with res-HTN (adjusted hazard ratio 1.18, 95\% CI 0.98-1.43). In the REACH registry, res-HTN, as compared with nonresistant HTN, was associated with increased allcause mortality (hazard ratio 1.11, 95\% CI 1.03-1.20), cardiovascular mortality (hazard ratio $1.17,95 \%$ CI 1.06-1.29), nonfatal MI (hazard ratio 1.23 , 95\% CI 1.07-1.40), and heart failure hospitalization (OR 1.49, 95\% CI 1.36-1.62), but not stroke, in patients with demonstrable atherosclerotic disease or those at risk of atherosclerotic disease [10]. Our results confirm these findings and suggest that a similar level of increased risk is associated with res-HTN in patients with concomitant CAD, although event rates are considerably greater in patients with CAD than those without CAD.

The exact mechanism by which res-HTN increases cardiovascular risk is unknown, but two plausible hypotheses are that patients with res-HTN have likely had a greater BP burden (i.e. more severe or prolonged elevations in BP) over time compared with patients with nonresistant HTN, and res-HTN is reflective of adverse processes (e.g. increased reninangiotensin system stimulation and aldosterone production, increased arterial stiffness, atherosclerotic disease) that have been linked with increased cardiovascular risk $[1,5]$. The latter hypothesis, in particular, may be favored by several recent lines of evidence from our study and others. First, we found no difference in outcomes comparing the patients with controlled res-HTN and uncontrolled res-HTN in our secondary analysis, despite an approximately $27 / 10 \mathrm{mmHg}$ difference in mean BP at the visit prior to an event or censoring. Second, in the REACH registry, a dose-response relationship was observed between an increased number of medications required and increasing cardiovascular risk, regardless of BP level, in patients with res-HTN [10]. Lastly, in a recent post-hoc analysis of the Valsartan Antihypertensive Long-Term Use Evaluation (VALUE) trial, a significantly greater risk of total MI, cardiovascular death, hospitalization for heart failure, and a composite of nonfatal stroke, nonfatal MI, or cardiovascular death, was observed in patients with nonresistant HTN who required combination therapy to control BP compared with those requiring only monotherapy to control BP [24]. Taken together, these findings suggest 
that for most major cardiovascular outcomes studied (save possibly stroke), the presence of res-HTN (or other adverse processes underlying res-HTN) is a more important prognostic factor than BP level or BP control. Alternatively, others have suggested that the use of a greater number of antihypertensive agents may not fully mitigate the long-term risks of elevated BP, possibly due to a J-curve phenomenon [10]. However, based on our results, this supposition seems unlikely as we excluded patients with BP below 130/80 mmHg.

A final noteworthy point is that we observed a differential effect of res-HTN on adverse outcomes. Specifically, res-HTN, compared with controlled HTN, was strongly associated with increased risk for all-cause mortality, cardiovascular mortality, and nonfatal stroke; however, an association with nonfatal MI was not observed. Although ours is not the first study to demonstrate a lack of association between res-HTN and greater risk of nonfatal MI $[9,10]$, the root cause of this differential effect on outcomes remains unexplored. One possibility may be that unknown factors underlying res-HTN may be associated with a differential effect on the noncardiac vasculature and organs [25]. These unknown factors could be more detrimental to the large systemic arteries and cerebral vessels, possibly contributing to widespread effects beyond acute events resulting from thrombotic occlusion due to coronary artery plaque rupture or erosion. Exaggerated arterial stiffening may be one such detrimental effect contributing to this differential influence on adverse outcomes [26]. Alternately, it is possible that MI in persons with res-HTN may be somehow different and may not meet the usual criteria for clinical diagnosis.

The major strengths of our study are the high-quality data, including blinded adjudication of outcomes, long follow-up, and the large sample size. However, our study has noteworthy limitations. First, our results are most generalizable to the growing population of patients with HTN and CAD, but these findings were obtained by a post-hoc analysis of a prospective randomized trial and thus our conclusions should be taken with a degree of caution. Second, although approximately $88 \%$ of res-HTN patients in the primary analysis were treated with a diuretic, our definition of res-HTN did not require use of these agents. However, we performed sensitivity analyses using the more narrow definition of res-HTN espoused by the European Society of Hypertension and European Society of Cardiology (i.e. requiring that a diuretic be included in the antihypertensive regimen [27]) in which we observed remarkably similar results to those from our primary analyses. Third, we did not have 24-h ambulatory BP monitoring (ABPM) data for all INVEST participants to differentiate those with isolated clinic BP elevations from those with concurrent clinic and ambulatory BP elevations. However, we did perform 24-h ABPM in a subset of INVEST patients and these ABPM data were generally consistent with clinic BP values (unpublished data), suggesting a relatively low prevalence of isolated clinic BP elevation in the INVEST study. Nevertheless, we cannot exclude the possibility that some patients may have been misclassified using only clinic BP values. Finally, secondary forms of hypertension were not assessed and medication adherence data were not collected in INVEST; thus, we cannot exclude the possibility that some patients with secondary or pseudoresistant hypertension were misclassified as having res-HTN. Likewise, patients with what amounts to res-HTN, but who were not treated with at least four antihypertensive agents, may have been misclassified as having uncontrolled HTN, which may have contributed to the lack of significant difference in outcomes between res-HTN and uncontrolled HTN groups. 
In conclusion, this post-hoc analysis from INVEST suggests that res-HTN is common in persons with CAD and that the presence of res-HTN portends an increased risk of major cardiovascular events and death, regardless of BP control. These findings have important implications for clinical practice and future research. Our observations reinforce the importance of recognizing patients with CAD at risk for res-HTN. Particular attention should be paid to those with comorbid cardiovascular disease, diabetes, and renal insufficiency, since these comorbidities are associated with the greatest risk of developing res-HTN. Additionally, our findings highlight the need for future studies to clarify the pathophysiologic cause(s) of increased cardiovascular risk in patients with res-HTN and to determine the most appropriate risk reduction strategies for these patients.

\section{Supplementary Material}

Refer to Web version on PubMed Central for supplementary material.

\section{Acknowledgments}

Sources of funding: INVEST was supported by the University of Florida Opportunity Fund and grants from Abbott Laboratories. The present research is supported, in part, by grants K23HL086558 and U01GM074492 from the National Institutes of Health R.M.C.DeH.

\section{References}

1. Calhoun DA, Jones D, Textor S, Goff DC, Murphy TP, Toto RD, et al. Resistant hypertension: diagnosis, evaluation, and treatment. A scientific statement from the American Heart Association Professional Education Committee of the Council for High Blood Pressure Research. Hypertension. 2008; 51:1403-1419. [PubMed: 18391085]

2. Cushman WC, Ford CE, Cutler JA, Margolis KL, Davis BR, Grimm RH, et al. Success and predictors of blood pressure control in diverse north american settings: The Antihypertensive and Lipid-Lowering Treatment to Prevent Heart Attack Trial (ALLHAT). J Clin Hypertens (Greenwich). 2002; 4:393-404. [PubMed: 12461301]

3. Gupta AK, Nasothimiou EG, Chang CL, Sever PS, Dahlöf B, Poulter NR. ASCOT Investigators. Baseline predictors of resistant hypertension in the Anglo-Scandinavian Cardiac Outcome Trial (ASCOT): a risk score to identify those at high-risk. J Hypertens. 2011; 29:2004-2013. [PubMed: 21881528]

4. Egan BM, Zhao Y, Axon RN, Brzezinski WA, Ferdinand KC. Uncontrolled and apparent treatment resistant hypertension in the United States, 1988 to 2008. Circulation. 2011; 124:1046-1058. [PubMed: 21824920]

5. Smith SM. Epidemiology, prognosis, and treatment of resistant hypertension. Pharmacotherapy. 2013; 33:1071-1086. [PubMed: 23722533]

6. Persell SD. Prevalence of resistant hypertension in the United States, 2003-2008. Hypertension. 2011; 57:1076-1080. [PubMed: 21502568]

7. Brambilla G, Bombelli M, Seravalle G, Cifkova R, Laurent S, Narkiewicz K, et al. Prevalence and clinical characteristics of patients with true resistant hypertension in central and Eastern Europe: data from the BP-CARE study. J Hypertens. 2013; 31:2018-2024. [PubMed: 23838657]

8. Pierdomenico SD, Lapenna D, Bucci A, Di Tommaso R, Di Mascio R, Manente BM, et al. Cardiovascular outcome in treated hypertensive patients with responder, masked, false resistant, and true resistant hypertension. Am J Hypertens. 2005; 18:1422-1428. [PubMed: 16280275]

9. Daugherty SL, Powers JD, Magid DJ, Tavel HM, Masoudi FA, Margolis KL, et al. Incidence and prognosis of resistant hypertension in hypertensive patients. Circulation. 2012; 125:1635-1642. [PubMed: 22379110] 
10. Kumbhani DJ, Steg PG, Cannon CP, Eagle KA, Smith SC, Crowley K, et al. Resistant hypertension: a frequent and ominous finding among hypertensive patients with atherothrombosis. Eur Heart J. 2013; 34:1204-1214. [PubMed: 23144048]

11. Lozano R, Naghavi M, Foreman K, Lim S, Shibuya K, Aboyans V, et al. Global and regional mortality from 235 causes of death for 20 age groups in 1990 and 2010: a systematic analysis for the Global Burden of Disease study 2010. Lancet. 2012; 380:2095-2128. [PubMed: 23245604]

12. Pepine CJ, Handberg EM, Cooper-DeHoff RM, Marks RG, Kowey P, Messerli FH, et al. A calcium antagonist vs. a noncalcium antagonist hypertension treatment strategy for patients with coronary artery disease. The international verapamil-trandolapril study (INVEST): a randomized controlled trial. JAMA. 2003; 290:2805-2816. [PubMed: 14657064]

13. Pepine CJ, Handberg-Thurmond E, Marks RG, Conlon M, Cooper-DeHoff R, Volkers P, Zellig P. Rationale and design of the international verapamil sr/trandolapril study (INVEST): an internetbased randomized trial in coronary artery disease patients with hypertension. J Am Coll Cardiol. 1998; 32:1228-1237. [PubMed: 9809930]

14. Chobanian AV, Bakris GL, Black HR, Cushman WC, Green LA, Izzo JL, et al. The Seventh Report of the Joint National Committee on Prevention, Detection, Evaluation, and Treatment of High Blood Pressure: the JNC 7 report. JAMA. 2003; 289:2560-2572. [PubMed: 12748199]

15. Messerli FH, Mancia G, Conti CR, Hewkin AC, Kupfer S, Champion A, et al. Dogma disputed: can aggressively lowering blood pressure in hypertensive patients with coronary artery disease be dangerous? Ann Intern Med. 2006; 144:884-893. [PubMed: 16785477]

16. Bakris G, Briasoulis A, Dahlof B, Jamerson K, Weber MA, Kelly RY, et al. Comparison of benazepril plus amlodipine or hydrochlorothia-zide in high-risk patients with hypertension and coronary artery disease. Am J Cardiol. 2013; 112:255-259. [PubMed: 23582626]

17. Dudenbostel T, Glasser SP. Effects of antihypertensive drugs on arterial stiffness. Cardiol Rev. 2012; 20:259-263. [PubMed: 22573107]

18. Williams B. Evaluating interventions to reduce central aortic pressure, arterial stiffness and morbidity-mortality. J Hypertens. 2012; 30(Suppl):S13-18. [PubMed: 23124100]

19. Williams B, Lacy PS, Thom SM, Cruickshank K, Stanton A, Collier D, et al. Differential impact of blood pressure-lowering drugs on central aortic pressure and clinical outcomes: principal results of the Conduit Artery Function Evaluation (CAFE) study. Circulation. 2006; 113:1213-1225. [PubMed: 16476843]

20. Morgan T, Lauri J, Bertram D, Anderson A. Effect of different anti-hypertensive drug classes on central aortic pressure. Am J Hypertens. 2004; 17:118-123. [PubMed: 14751652]

21. Paulis L, Simko F. Blood pressure modulation and cardiovascular protection by melatonin: potential mechanisms behind. Physiol Res. 2007; 56:671-684. [PubMed: 18197748]

22. Kario K. Are melatonin and its receptor agonist specific antihypertensive modulators of resistant hypertension caused by disrupted circadian rhythm? J Am Soc Hypertens. 2011; 5:354-358. [PubMed: 21592880]

23. Scheer FA, Van Montfrans GA, van Someren EJ, Mairuhu G, Buijs RM. Daily nighttime melatonin reduces blood pressure in male patients with essential hypertension. Hypertension. 2004; 43:192-197. [PubMed: 14732734]

24. Weber MA, Julius S, Kjeldsen SE, Jia Y, Brunner HR, Zappe DH, et al. Cardiovascular outcomes in hypertensive patients: comparing single-agent therapy with combination therapy. J Hypertens. 2012; 30:2213-2222. [PubMed: 23011525]

25. Esler M. The 2009 Carl Ludwig Lecture: pathophysiology of the human sympathetic nervous system in cardiovascular diseases: the transition from mechanisms to medical management. J Appl Physiol. 2010; 108:227-237. [PubMed: 19940096]

26. Brandt MC, Reda S, Mahfoud F, Lenski M, Bohm M, Hoppe UC. Effects of renal sympathetic denervation on arterial stiffness and central hemodynamics in patients with resistant hypertension. J Am Coll Cardiol. 2012; 60:1956-1965. [PubMed: 23062529]

27. Mancia G, Fagard R, Narkiewicz K, Redon J, Zanchetti A, Böhm M, et al. 2013 ESH/ESC Guidelines for the management of arterial hypertension. Eur Heart J. 2013; 34:2159-2219. [PubMed: 23771844] 


\section{Abbreviations}

$\begin{array}{ll}\text { BP } & \text { blood pressure } \\ \text { CAD } & \text { coronary artery disease } \\ \text { INVEST } & \text { INternational VErapamil SR-Trandolapril Study } \\ \text { res-HTN } & \text { resistant hypertension }\end{array}$




\section{Reviewers' Summary Evaluations}

\section{Reviewer 1}

The study is strengthened by its large sample size, longitudinal design, and rigorous adjudication of events. Studyweaknesses include having not distinguished apparent from true resistant hypertension with exclusion of whitecoat resistant hypertension and poor medication adherence.

\section{Reviewer 2}

\section{Strengths}

On a large cohort of patients with CAD, this work shows in a very clear and convincing way the large prevalence of resistant hypertension and its impact in terms of adverse outcomes. This posthoc analysis of the INVEST trial has been well performed and highlights the huge work we still have to do to improve blood pressure control especially in this population of $\mathrm{CAD}$ who already experienced a cardiovascular event.

\section{Weaknesses}

As a posthoc analysis, these results have to be confirmed. 


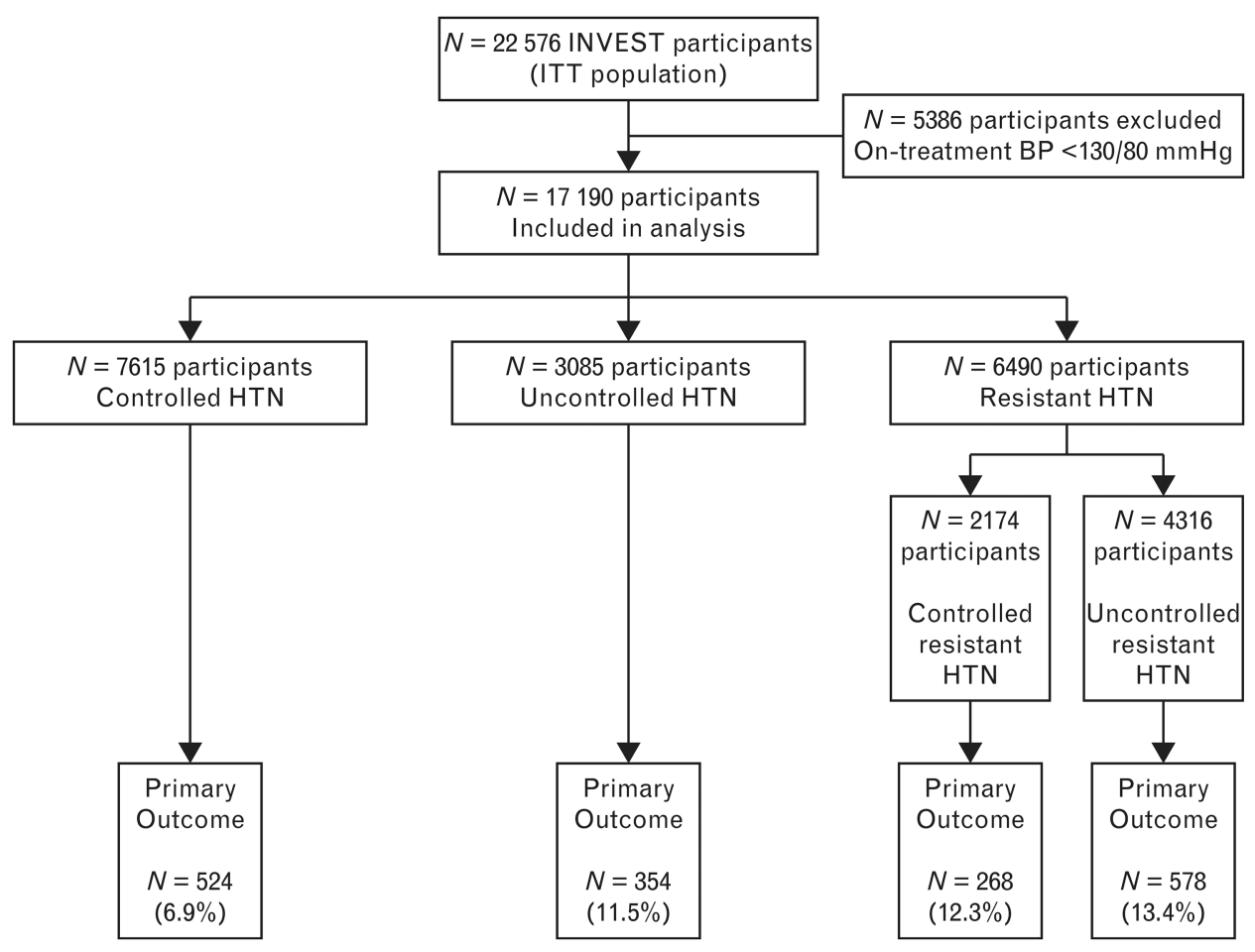

Figure 1.

Flow diagram of participants included in this study. Controlled HTN indicates BP below $140 / 90 \mathrm{mmHg}$ on three or fewer antihypertensive drugs. Res-HTN indicates BP at least $140 / 90 \mathrm{mmHg}$ on at least three antihypertensive drugs (uncontrolled res-HTN) or BP below 140/90 on at least four antihypertensive drugs (controlled res-HTN). Uncontrolled HTN indicates BP at least 140/90 $\mathrm{mmHg}$ on two or fewer antihypertensive drugs. BP, blood pressure; ITT, intention to treat; res-HTN, resistant hypertension. 

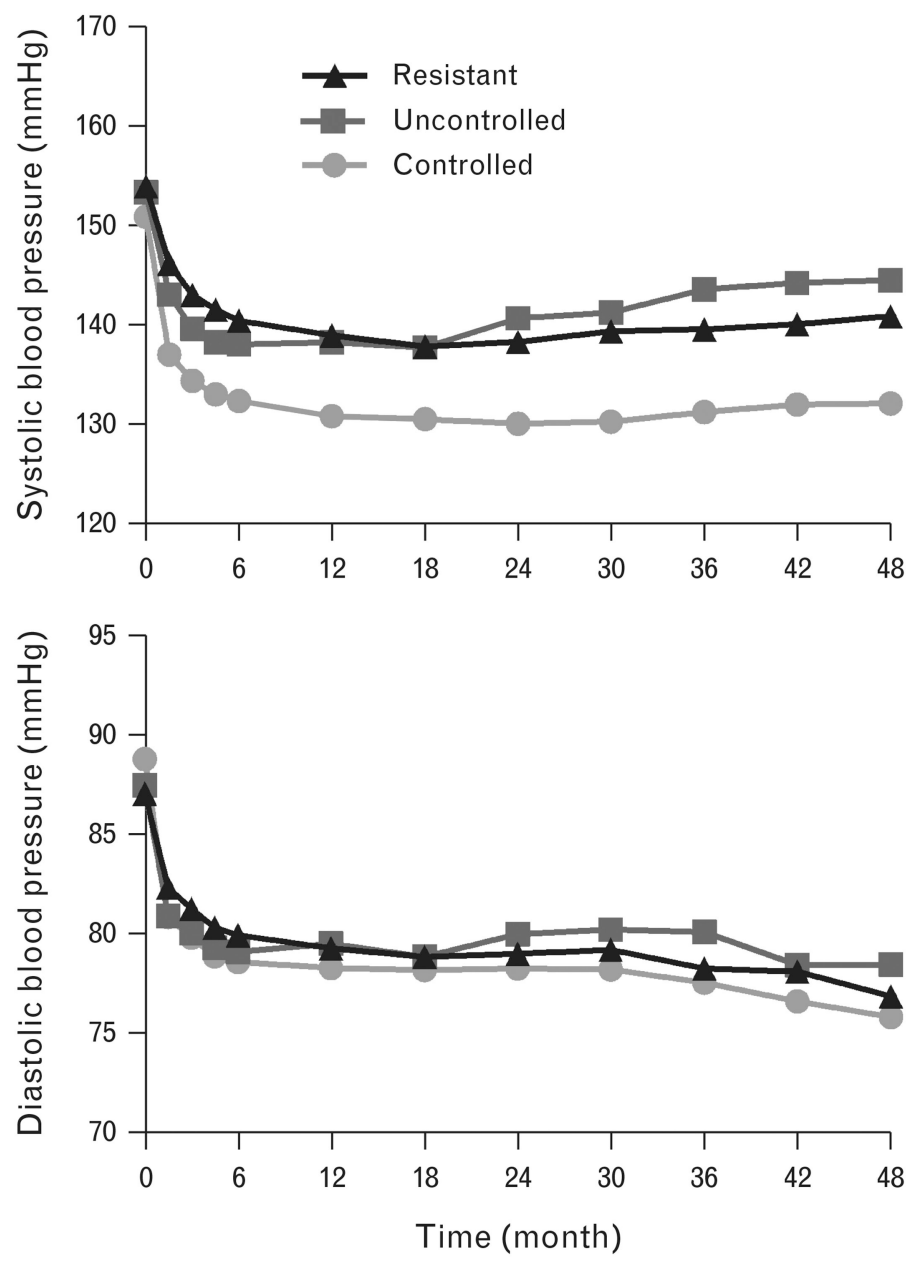

Figure 2.

Mean SBP (top panel) and DBP (bottom panel) according to hypertension control group and study follow-up. 


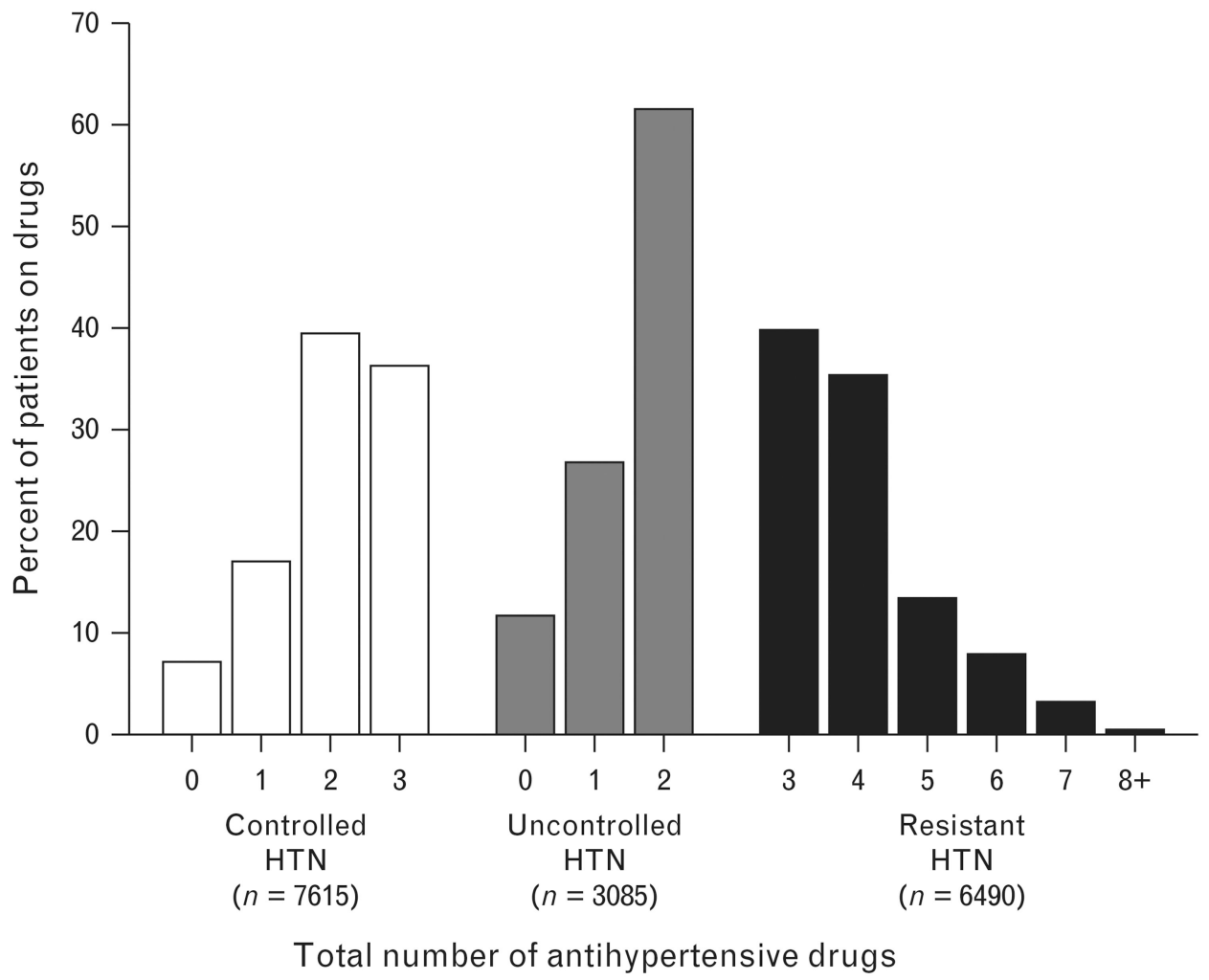

Figure 3.

Percentage of patients according to total (study + nonstudy) number of antihypertensive drugs and hypertension control group. 


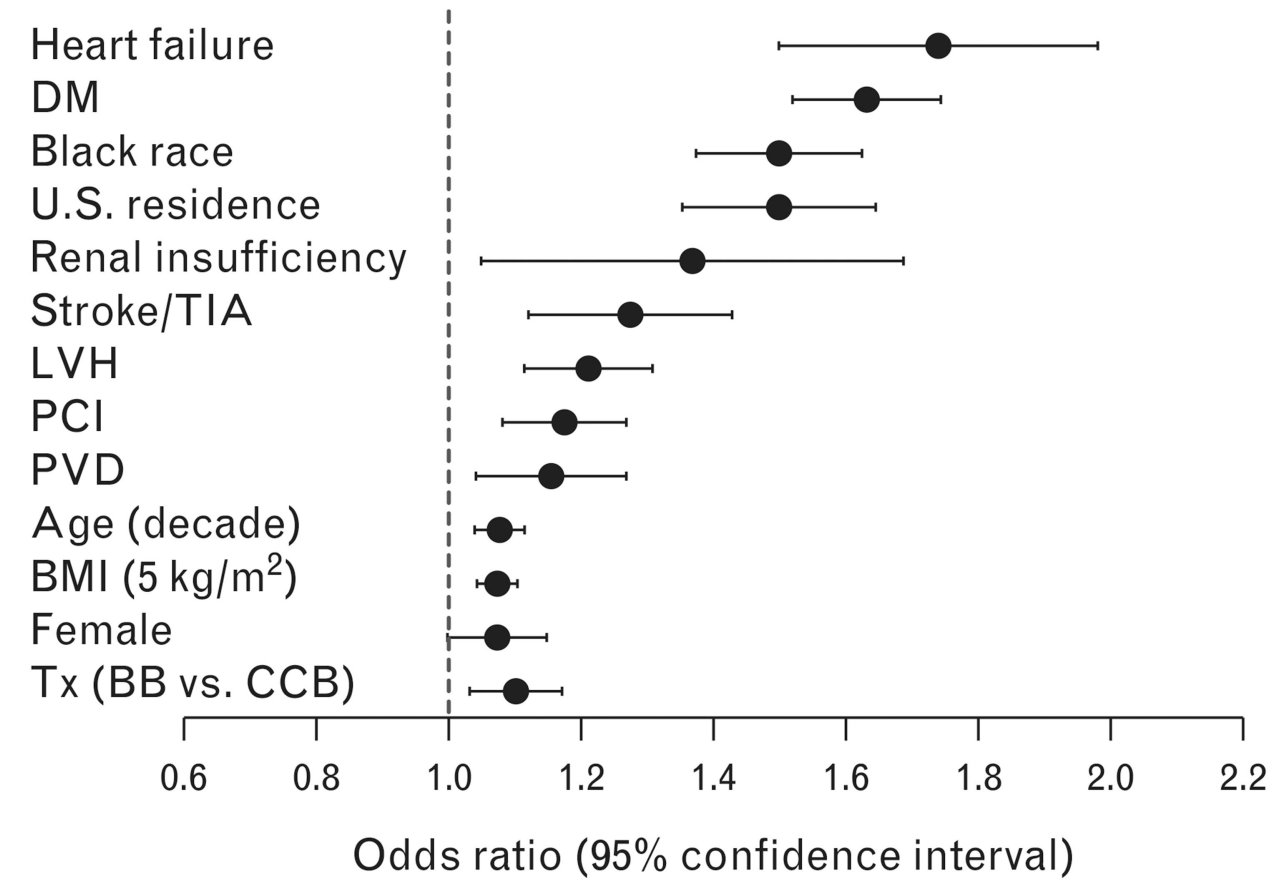

Figure 4.

Baseline predictors of increased risk for resistant hypertension. BB, $\beta$-blocker; $\mathrm{CCB}$, calcium channel blocker; DM, diabetes mellitus; LVH, left-ventricular hypertrophy; PAD, peripheral artery disease; PCI, percutaneous coronary intervention; PVD, peripheral vascular disease; TIA, transient ischemic attack; Tx, treatment. 


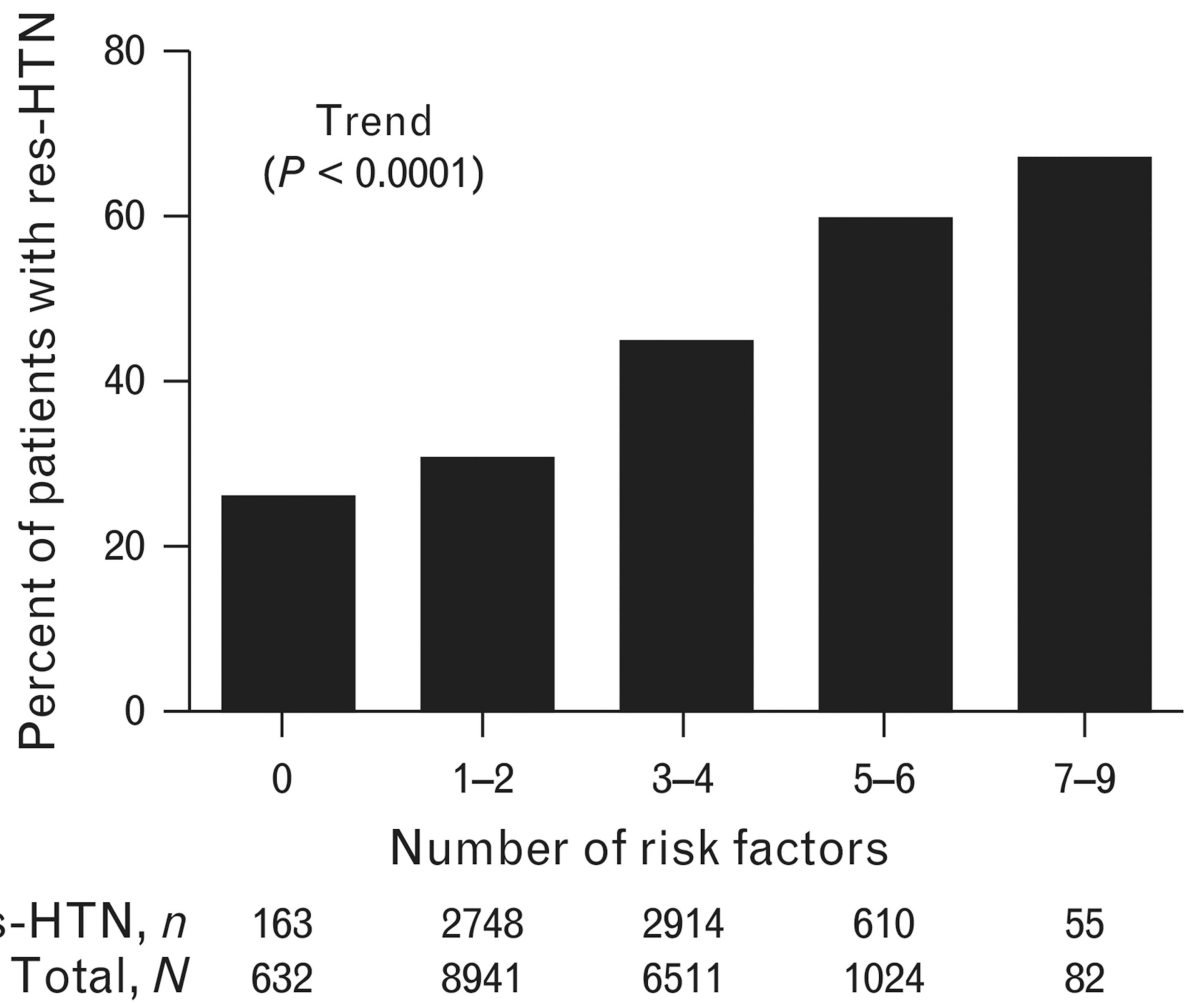

Figure 5.

Prevalence of resistant hypertension according to total number of risk factors present. Risk factors represent those identified as independent baseline predictors of risk for resistant hypertension in multivariate logistic regression analyses and include presence of heart failure, diabetes, Black race, US residence, renal insufficiency, stroke/TIA, left-ventricular hypertrophy, peripheral artery disease, percutaneous coronary intervention, age, BMI, female sex, and treatment with $\beta$-blocker strategy. res-HTN, resistant hypertension; TIA, transient ischemic attack. 


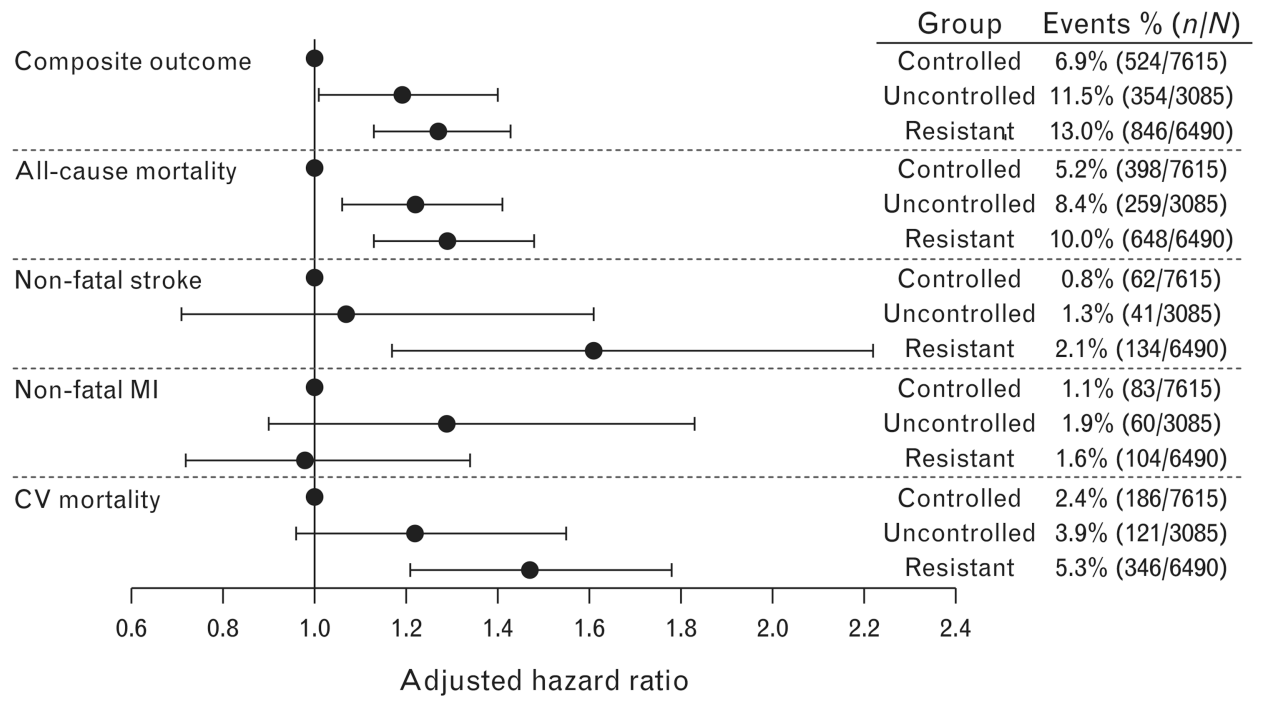

Figure 6.

Rates and adjusted risk for composite and individual adverse outcomes risk according to hypertension control group. Composite outcome represents first occurrence of all-cause mortality, nonfatal stroke, or nonfatal MI. MI, myocardial infarction. 


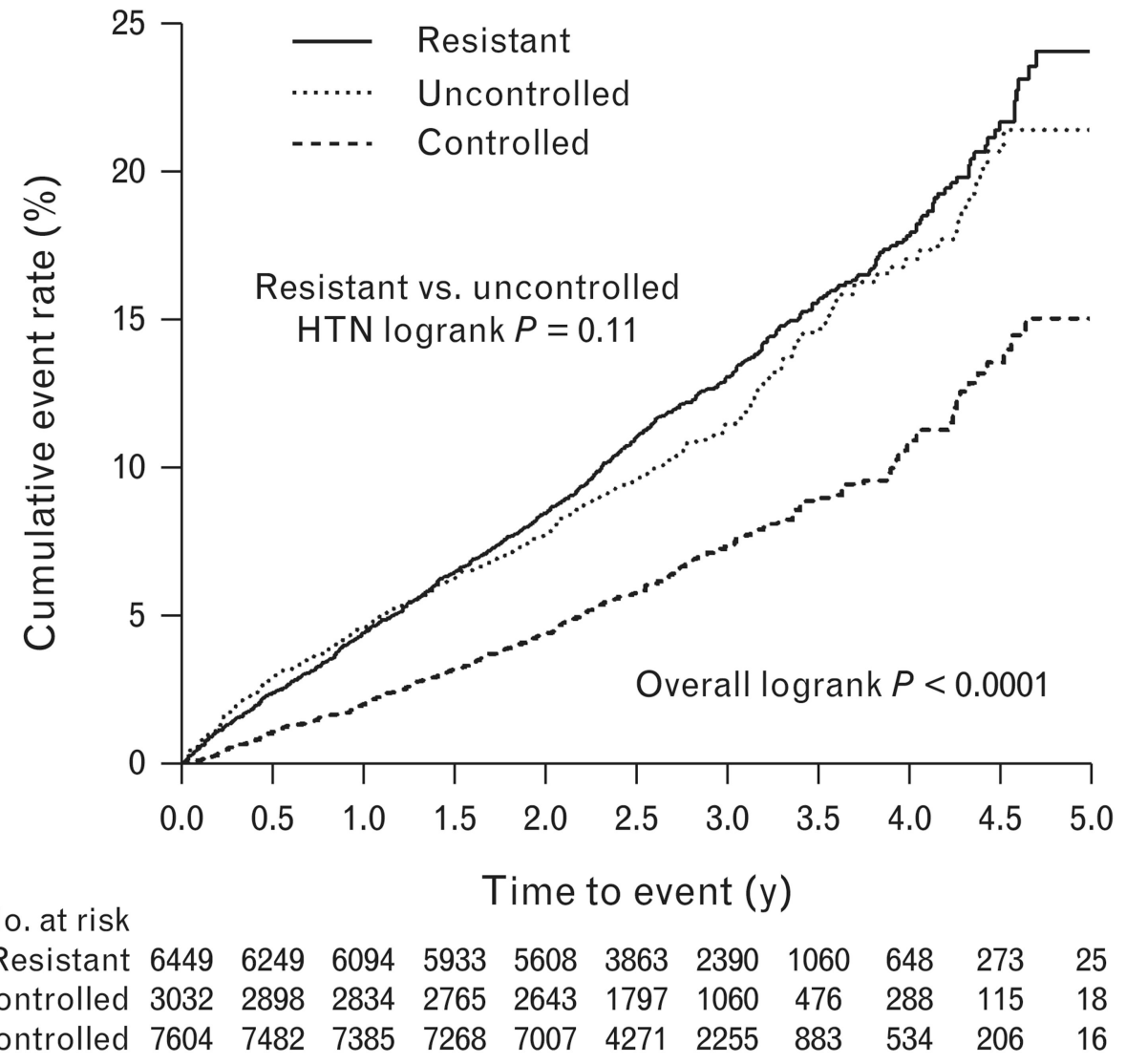

Figure 7.

Cumulative event rate for the primary outcome according to hypertension control group. 
Table 1

Baseline characteristics according to hypertension control group

\begin{tabular}{|c|c|c|c|c|}
\hline Variable & Controlled $(n=7615)$ & Uncontrolled ( $n=3085$ ) & Resistant $(n=6490)$ & $P$ value \\
\hline Mean age \pm SD (years) & $65.2 \pm 9.5$ & $66.6 \pm 9.9$ & $66.4 \pm 9.8$ & $<0.0001$ \\
\hline Age $>70$ years & 29.9 & 35.7 & 34.9 & $<0.0001$ \\
\hline Women & 52.0 & 53.2 & 54.0 & 0.048 \\
\hline Race/ethnicity & & & & $<0.0001$ \\
\hline White & 46.2 & 52.7 & 49.0 & \\
\hline Black & 11.3 & 13.6 & 20.1 & \\
\hline Hispanic & 40.0 & 30.6 & 28.5 & \\
\hline Mean BMI $\pm \mathrm{SD}\left(\mathrm{kg} / \mathrm{m}^{2}\right)$ & $29.1 \pm 9.0$ & $29.0 \pm 5.6$ & $30.0 \pm 6.4$ & $<0.0001$ \\
\hline Mean $\mathrm{SBP} \pm \mathrm{SD}(\mathrm{mmHg})$ & $151 \pm 19$ & $153 \pm 19$ & $154 \pm 20$ & $<0.0001$ \\
\hline Mean $\mathrm{DBP} \pm \mathrm{SD}(\mathrm{mmHg})$ & $89 \pm 12$ & $88 \pm 12$ & $87 \pm 12$ & $<0.0001$ \\
\hline \multicolumn{5}{|l|}{ History of } \\
\hline MI & 29.5 & 31.2 & 33.2 & $<0.0001$ \\
\hline Angina & 69.8 & 65.7 & 66.0 & $<0.0001$ \\
\hline Revascularization & 23.1 & 27.2 & 29.4 & $<0.0001$ \\
\hline Stroke/TIA & 6.3 & 6.2 & 9.3 & $<0.0001$ \\
\hline $\mathrm{LVH}$ & 22.1 & 18.0 & 24.3 & $<0.0001$ \\
\hline Heart failure (class I-III) & 4.1 & 3.3 & 8.1 & $<0.0001$ \\
\hline PAD & 10.0 & 11.6 & 13.9 & $<0.0001$ \\
\hline Smoking (ever) & 45.8 & 44.1 & 45.9 & 0.22 \\
\hline Diabetes $^{a}$ & 23.6 & 25.0 & 36.0 & $<0.0001$ \\
\hline Renal impairment ${ }^{b}$ & 1.1 & 1.9 & 2.7 & $<0.0001$ \\
\hline US residency & 69 & 81 & 81 & $<0.0001$ \\
\hline \multicolumn{5}{|l|}{ Medications } \\
\hline Any lipid-lowering agent & 34.6 & 33.4 & 37.0 & $<0.0001$ \\
\hline Nitrate & 36.4 & 29.4 & 36.1 & $<0.0001$ \\
\hline Aspirin & 55.8 & 51.6 & 57.1 & $<0.0001$ \\
\hline NSAID & 17.3 & 15.6 & 19.4 & $<0.0001$ \\
\hline Potassium supplement & 4.0 & 5.4 & 11.3 & $<0.0001$ \\
\hline Any antidiabetic agent ${ }^{c}$ & 19.1 & 19.7 & 28.1 & $<0.0001$ \\
\hline
\end{tabular}

Numbers represent percentage unless otherwise specified.

LVH, left-ventricular hypertrophy; MI, myocardial infarction; PAD, peripheral artery disease.

${ }^{a}$ History of or currently taking antidiabetic agent at baseline.

${ }^{b}$ History of or currently have elevated serum creatinine level but less than $4 \mathrm{mg} / \mathrm{dl}(<354 \mu \mathrm{mol} / \mathrm{l})$.

J Hypertens. Author manuscript; available in PMC 2015 March 01. 
${ }^{c}$ Both insulin and oral hypoglycemics, or either of the two. 
